Spect r oel ect r ochemi cal char act er i zat i on of dendri mer-por phyri $n$ associ at es at pol arized I i qui d I I qui d i nt erfaces

\begin{tabular}{|l|l|}
\hline 著者 & $\begin{array}{l}\text { Sakae H r oki, Nagat ani H rohi sa, Nor i t a } \\
\text { Kot ar o, I mur a Hi sanor i }\end{array}$ \\
\hline $\begin{array}{l}\text { j our nal or } \\
\text { publ i cat i on ti tl e }\end{array}$ & Langmi r \\
\hline vol une & 30 \\
\hline nunber & 3 \\
\hline page r ange & $937-945$ \\
\hline year & 2014 01- 28 \\
\hline URL & ht t p: //hdl . handl e. net /2297/36905 \\
\hline
\end{tabular}




\title{
Spectroelectrochemical Characterization of
}

\author{
Dendrimer-Porphyrin Associates
}

\section{at Polarized Liquid|Liquid Interfaces}

Hiroki Sakae, ${ }^{\dagger}$ Hirohisa Nagatani, ${ }^{*}{ }^{\ddagger}$ Kotaro Morita, ${ }^{\ddagger}$ Hisanori Imura ${ }^{\ddagger}$

${ }^{\dagger}$ Division of Material Sciences, Graduate School of Natural Science and Technology,

Kanazawa University, Kakuma, Kanazawa 920-1192, Japan

${ }^{\ddagger}$ Faculty of Chemistry, Institute of Science and Engineering, Kanazawa University, Kakuma,

Kanazawa 920-1192, Japan

*To whom correspondence should be addressed: H. Nagatani

Tel: +81 76264 5692; Fax: +81 762646059

E-mail:nagatani@se.kanazawa-u.ac.jp 


\section{Abstract}

Molecular encapsulation of anionic porphyrins in $\mathrm{NH}_{2}$-terminated polyamidoamine (PAMAM) dendrimers and the interfacial behavior of the dendrimer-porphyrin associates were studied at the polarized water|1,2-dichloroethane (DCE) interface. The formation of the ion associates was significantly dependent on the $\mathrm{pH}$ condition and on the generation of dendrimers. 5,10,15,20Tetrakis(4-sulfonatophenyl)porphyrin (ZnTPPS ${ }^{4-}$ ) associated with the positively charged fourth generation (G4) PAMAM dendrimer was highly stabilized in the acidic aqueous solution without protolytic demetalation in a wide range of $\mathrm{pH}(\mathrm{pH}>2)$. In contrast to the zinc(II) complex, the free base porphyrin ( $\mathrm{H}_{2} \mathrm{TPPS}^{4-}$ ) was readily protonated under acidic conditions even in the presence of the dendrimers. In addition, the J-aggregates of diprotonated species, $\left(\mathrm{H}_{4} \mathrm{TPPS}^{2-}\right)_{\mathrm{n}}$, were preferably formed on the dendrimer. The interfacial mechanism of the dendrimer-porphyrin associates was analyzed in detail by potential modulated fluorescence (PMF) spectroscopy. PMF results indicated that the dendrimers incorporating porphyrin molecules were transferred across the positively polarized water|DCE interface via adsorption step, whereas the transfer responses of the porphyrin ions released from the dendrimers were observed at negatively polarized conditions. A negative shift of the transfer potential of porphyrin ions compared to the intrinsic transfer potential was apparently observed for each ion association system. The ion association stability between the dendrimer and the porphyrin molecules could be estimated from a negative shift of the transfer potential. ZnTPPS ${ }^{4-}$ exhibited relatively strong interaction with the higher generation dendrimer, whereas $\mathrm{H}_{2} \mathrm{TPPS}^{4-}$ was less effectively associated with the dendrimers. 


\section{Introduction}

An interface between two immiscible electrolyte solutions (ITIES) is two-dimensional specific reaction fields, where charge transfer processes and heterogeneous reaction could be controlled as a function of the Galvani potential difference between two liquid phases. ${ }^{1}$ The interfacial reaction system has been studied extensively for analytical sciences, synthesis of thin-layer materials, and biochemical applications..$^{2-4}$ The ion-partitioning property of species under electrochemical control is particularly useful to evaluate pharmacokinetic distribution of drugs. Ion transfer voltammetry and related electrochemical techniques allow us to access fundamental information on the charge transfer reaction. The surface sensitive spectroelectrochemical techniques such as potential modulation spectroscopy can provide deeper insights of the interfacial mechanism. ${ }^{5,6}$

Dendrimers are unique and nontraditional polymers with a well-defined macromolecular architecture consisting of a core, iterative branch units, and terminal groups. ${ }^{7,8}$ The reactivity of the dendrimer can chemically be modified by choosing a variety of functional groups in the periphery moiety. Various organic molecules and metal ions have been reported to be accommodated into the internal cavity and captured on the peripheral region of the dendrimer by hydrophobic and electrostatic interactions. ${ }^{9,10}$ The dendrimers have been examined as molecular capsule and light-emitting devices by hybridizing with dye molecules or metal nanoparticles. ${ }^{11-13}$ In particular, the potential application for drug delivery systems (DDS) has been attracted much attention. $^{14,15}$ The most widely studied polyamidoamine (PAMAM) dendrimer is constructed based on an ethylenediamine core and amino (full generation) or carboxyl (half generation) terminal groups. The net charge and conformation of the PAMAM dendrimer are significantly affected by the $\mathrm{pH}$ condition owing to the protonation equilibrium of terminal groups and tertiary 
amines in the interior. In addition, the molecular configuration of the PAMAM dendrimer tends to be a spherical shape from a flat-elliptical according to the increase of the generation. The molecular encapsulation features of a guest molecule thus could be affected by the generation of the dendrimer. The ion association and transfer mechanisms of the dendrimer at polarized liquid|liquid interfaces are very important to estimate a capability of the dendrimer in DDS and separation sciences. Recently, the ion transfer mechanism of the PAMAM dendrimer incorporating a fluorescence probe was studied in detail at the polarized water|1,2-dichloroethane (DCE) interface. The spectroelectrochemical analysis demonstrated that the amino-terminated fourth generation (G4) PAMAM dendrimer was transferred across the interface accompanied by the adsorption process. ${ }^{16}$ The molecular encapsulation of 8-anilino-1-naphthalenesulfonate (ANS ${ }^{-}$) and its bimolecular derivative (bis- $\mathrm{ANS}^{2-}$ ) in the carboxylate-terminated G3.5 PAMAM dendrimer was also investigated at the water|DCE interface as a function of $\mathrm{pH}$ and the Galvani potential difference. ${ }^{17}$ A stable encapsulation of bis-ANS ${ }^{2-}$ compared with the monomeric ANS $^{-}$ was explained by the electrostatic two-point interaction of anionic sulfonate groups with protonated tertiary amines in the interior. In addition, the electrostatic interaction between the G3.5 PAMAM dendrimer and bis-ANS ${ }^{2-}$ could be estimated quantitatively from a negative shift of the transfer potential of bis-ANS with respect to the intrinsic formal transfer potential.

Porphyrins have been extensively studied as biomimetic reactants, dye sensitizers, catalysts, spectrophotometric reagents and so on. ${ }^{18}$ The physicochemical property and reactivity of porphyrin compounds strongly depend on peripheral substituted groups as well as metal center. The porphyrins and metalloporphyrins, however, are readily protonated (via demetalation for metalloporphyrins) in acidic solutions inducing considerable spectral changes. ${ }^{19}$ On the other hand, the molecular size and net charge of the free base porphyrin are identical to its divalent 
metal complex. The porphyrin molecules are therefore suitable for a contrastive evaluation of the contribution of the metal center in molecular encapsulation systems.

In this study, the molecular encapsulation behavior of the free base and zinc(II) complex of water-soluble porphyrin, 5,10,15,20-tetrakis(4-sulfonatophenyl)porphyrin (TPPS) in the aminoterminated G4 and G2 PAMAM dendrimers was studied at the polarized water|DCE interface by potential modulated fluorescence (PMF) spectroscopy. ZnTPPS ${ }^{4-}$ and $\mathrm{H}_{2} \mathrm{TPPS}^{4-}$ were effectively associated with the positively charged PAMAM dendrimer depending on $\mathrm{pH}$ and the Galvani potential difference. The stabilization of porphyrin molecules in the PAMAM dendrimer could be estimated from the apparent transfer potential of the porphyrins.

\section{Experimental Section}

2.1. Reagents. The G4 and G2 PAMAM dendrimers with ethylenediamine core were purchased from Aldrich (10 and $20 \mathrm{wt} \%$ in methanol) and prepared as an aqueous solution after removing methanol by drying in ultrapure argon (> 99.999\%). The anionic porphyrins, 5,10,15,20tetrakkis(4-sulfonatophenyl)porphyrin $\quad\left(\mathrm{H}_{2} \mathrm{TPPS}^{4-}\right.$ ) disulfuric acid tetrahydrate (Dojindo Laboratories) and the tetrasodium salt of its zinc(II) complex (Na4ZnTPPS) (Frontier Scientific/Porphyrin Products), were used as received. The composition of the electrochemical cell was represented in Figure 1. The concentration of the dendrimers and the porphyrins was $1.0 \times 10^{-5} \mathrm{~mol} \mathrm{dm}{ }^{-3}$. The supporting electrolytes were $1.0 \times 10^{-2} \mathrm{~mol} \mathrm{dm}^{-3} \mathrm{LiCl}$ for the aqueous phase and $5.0 \times 10^{-3}$ mol $\mathrm{dm}^{-3}$ bis(triphenylphosphoranylidene)ammonium tetrakis(pentafluorophenyl)borate (BTPPATPFB) for the organic phase, respectively. BTPPATPFB was prepared by metathesis of bis(triphenylphosphoranylidene)ammonium 
chloride (BTPPACl) (Aldrich, > 97\%) and lithium tetrakis(pentafluorophenyl)borate ethyl ether complex (TCI, $\geq 70 \%$ ). The organic solvent, 1, 2-dichloroethane (DCE), was of HPLC grade (Nacalai Tesque, $>99.7 \%$ ). All other reagents were of the highest grade available and used without further purification. The aqueous solutions were prepared with purified water from a Milli-Q system (Millipore Simpli Lab-UV/Direct-Q 3 UV). The pH of the aqueous phase was controlled by the addition of $\mathrm{HCl}$ for acidic conditions and $\mathrm{LiH}_{2} \mathrm{PO}_{4} / \mathrm{LiOH}$ buffer and $\mathrm{LiOH}$ for neutral and alkaline conditions, respectively.

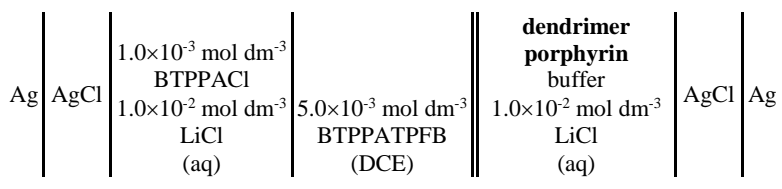

Figure 1. Composition of the electrochemical cell.

2.2. Apparatus. The spectroelectrochemical cell used in all measurements was analogous to the one previously reported. ${ }^{5}$ The water|DCE interface with a geometrical area of $0.50 \mathrm{~cm}^{2}$ was polarized by a four-electrode potentiostat (Hokuto Denko HA1010 mM1A). Platinum wires were used as counter electrodes in both aqueous and organic phases. The Luggin capillaries were provided for the reference electrodes $(\mathrm{Ag} / \mathrm{AgCl})$ in both phases. The Galvani potential difference ( $\Delta_{\mathrm{o}}^{\mathrm{w}} \phi \equiv \phi^{\mathrm{w}}-\phi^{\mathrm{o}}$ ) was estimated by taking the formal transfer potential ( $\Delta_{\mathrm{o}}^{\mathrm{w}} \phi^{\mathrm{o}^{\prime}}$ ) of tetramethylammonium as $0.160 \mathrm{~V} .{ }^{20} \mathrm{UV}$-Vis absorption and fluorescence spectra of the aqueous solution were measured by a UV-Vis spectrophotometer (JASCO, V-630) and a fluorescence spectrophotometer (Hitachi, F-2500), respectively. The fluorescence spectrum was taken at an excitation maximum in each system.

2.3. Potential Modulated Fluorescence Spectroscopy. The water|DCE interface under electrochemical control was illuminated from the organic phase by a cw laser diode of $50 \mathrm{~mW}$ at 
$404 \mathrm{~nm}$ (Coherent, CUBE 405-50C) in total internal reflection (TIR) mode. The angle of incidence $(\psi)$ of the $p$-polarized laser beam was set as ca. $75^{\circ}$. The fluorescence emitted from the interfacial region was collected perpendicularly to the interface by an optical fiber fitted to a photomultiplier tube through a monochromator (Shimadzu, SPG-120S). The ac modulated fluorescence signal at $1 \mathrm{~Hz}$ was analyzed as a function of ac potential modulation by a digital lock-in amplifier (NF LI5640) for potential dependence measurements at $5 \mathrm{mV} \mathrm{s}^{-1}$, while a frequency response analyzer (NF FRA5022) was employed for frequency dependence measurements from 0.1 to $100 \mathrm{~Hz}$. Further details of the PMF setup and analytical procedure are described elsewhere. ${ }^{5,21,22}$ All of the experiments were carried out in a thermostated room at $298 \pm 2 \mathrm{~K}$.

\section{Results and Discussion}

\subsection{Ion Association Behavior between PAMAM Dendrimers and Anionic Porphyrins in the}

Aqueous Solution. The intermolecular interaction between the dendrimers and the anionic porphyrins in the aqueous phase was investigated at various pHs. Figure 2(a) shows UV-Vis absorption and fluorescence spectra of ZnTPPS ${ }^{4-}$ in the absence and presence of equimolar dendrimer at $\mathrm{pH}$ 7.2. The absorption maximum wavelength at the Soret band $(421 \mathrm{~nm})$ of ZnTPPS $^{4-}$ was significantly red-shifted to $428 \mathrm{~nm}$ and $427 \mathrm{~nm}$, respectively, in the presence of the G4 and G2 PAMAM dendrimers. The corresponding red-shifts were also observed in the fluorescence spectra. The spectral changes demonstrate the effective ion association between the cationic dendrimer and the anionic porphyrin in the aqueous phase. In addition, the red-shift of the G4 PAMAM dendrimer system was larger than that of the G2 PAMAM dendrimer. These 

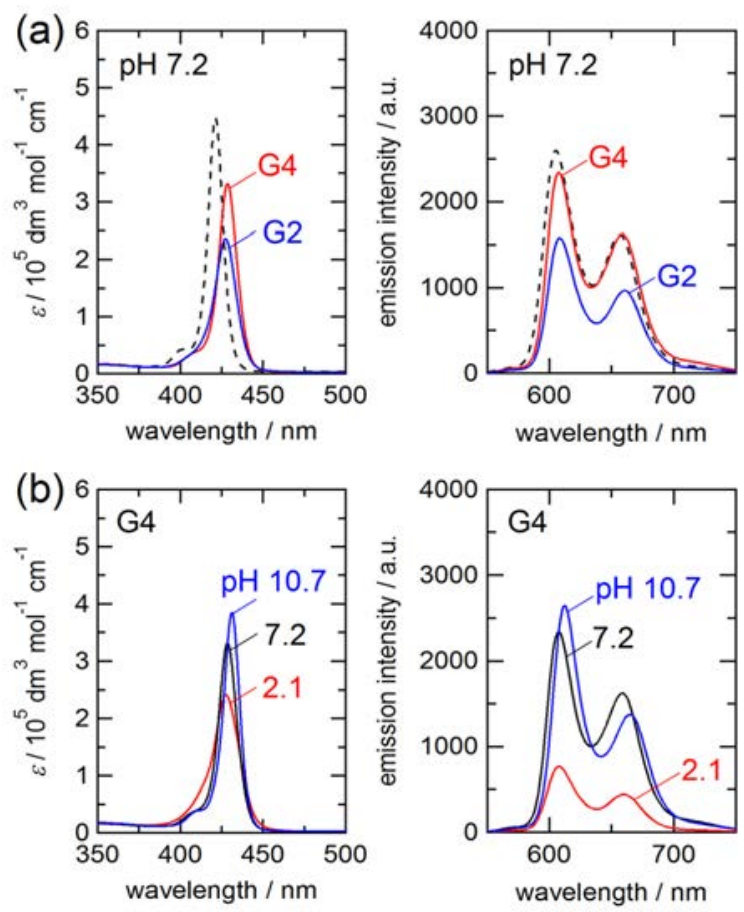

Figure 2. UV-Vis absorption and florescence spectra of $\mathrm{ZnTPPS}^{4-}$ in the presence of equimolar dendrimers in the aqueous solution. (a) The spectra measured in the G4 and G2 PAMAM dendrimer systems at $\mathrm{pH}$ 7.2. (b) The $\mathrm{pH}$ dependences of the spectra in the presence of the G4 PAMAM dendrimer. The dashed lines are the spectra measured in the absence of the dendrimer. The concentration of ZnTPPS ${ }^{4-}$ and the dendrimer was $1.0 \times 10^{-6} \mathrm{~mol} \mathrm{dm}^{-3}$.

spectral shifts would indicate that the encapsulation environment of the porphyrin molecules such as hydration and/or axial coordination is not identical in the G4 and G2 PAMAM dendrimer systems, in which the higher generation dendrimer seems to isolate ZnTPPS ${ }^{4-}$ from homogeneous solution phase. The G4 PAMAM dendrimer is regarded as spherical shape with diameters reported between 4.3 and $4.9 \mathrm{~nm}$ under various conditions, while the G2 PAMAM dendrimer is flat-elliptical shape with a diameter around $\sim 2.9 \mathrm{~nm}^{8,23} \mathrm{ZnTPPS}^{4-}$, whose longest diagonal axes are $\sim 1.9 \mathrm{~nm},{ }^{24}$ could be penetrated into the hydrophobic interior of the G4 
PAMAM dendrimer in terms of the molecular dimension. On the other hand, the ZnTPPS ${ }^{4-}$ molecules associated with the small G2 PAMAM dendrimer is exposed to the solution phase in any molecular arrangement. The change of solvation structure of ZnTPPS ${ }^{4-}$ would be enhanced with the G4 PAMAM dendrimer. Figure 2(b) shows the $\mathrm{pH}$ dependence of the spectra of ZnTPPS $^{4-}$ in the presence of equimolar G4 PAMAM dendrimer in the aqueous solution. The spectra measured at $\mathrm{pH} 10.7$ were hardly modified from those of $\mathrm{pH}$ 7.2. The net charge on the G4 PAMAM dendrimer depends on protonation equilibria of 64 primary amines in the periphery moiety $\left(\mathrm{p} K_{\mathrm{a}, 1}: 9.20\right)$ and 62 tertiary amines as branch points $\left(\mathrm{p} K_{\mathrm{a}, 2}: 6.65\right) .{ }^{25}$ The G4 PAMAM dendrimer thus has only few positive charges, $\sim+2$, at $\mathrm{pH} 10.7$. The apparent spectral changes of ZnTPPS $^{4-}$ in the alkaline condition exhibit that the ion association between the anionic porphyrin and the dendrimer is promoted not only by the electrostatic interaction. Under acidic conditions, ZnTPPS $^{4-}$ was demetalated and protonated to the diacid form $\left(\mathrm{H}_{4} \mathrm{TPPS}^{2-}\right)$ in the absence of the dendrimer. $\mathrm{H}_{4} \mathrm{TPPS}^{2-}$ showed the Soret band at $434 \mathrm{~nm}$ and the Q bands at 645 and $594 \mathrm{~nm}$ (Supporting Information (SI): Figure S1). The spectra taken at $\mathrm{pH} 2.1$ indicated that ZnTPPS $^{4-}$ was effectively stabilized in the acidic aqueous solution by associating with the dendrimer. The broadening of the Soret band accompanied by relative decreases of the fluorescence intensity at $\mathrm{pH}$ 2.1, however, might be indicative of slight demetalation of ZnTPPS $^{4-}$. The similar stability enhancement of ZnTPPS ${ }^{4-}$ was also reported in the electrostatic formation of complex micelles with poly(ethyleneglycol)- $b$-poly(4-vinylpyridine) (PEG- $b$ P4VP) by Wang et al. ${ }^{26}$ At pH 2.1, the G4 PAMAM dendrimers exist as fully protonated cationic species in the aqueous solution. The considerable decrease in the fluorescence intensity at $\mathrm{pH} 2.1$ could thus be associated with the fluorescence quenching by the favorable electrostatic 

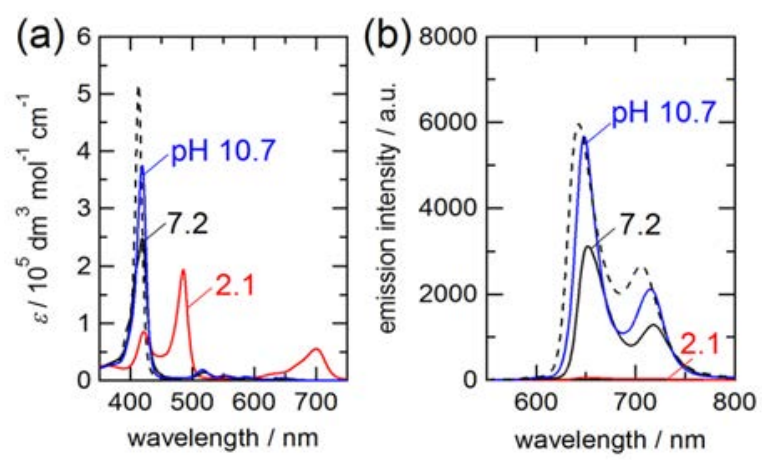

Figure 3. (a) UV-Vis absorption and (b) florescence spectra of $\mathrm{H}_{2} \mathrm{TPPS}^{4-}$ in the presence of equimolar G4 PAMAM dendrimer in the aqueous solution. The dashed lines are the spectra measured in the absence of the dendrimer at $\mathrm{pH}$ 7.2. The concentration of $\mathrm{H}_{2} \mathrm{TPPS}^{4-}$ and the dendrimer was $1.0 \times 10^{-6} \mathrm{~mol} \mathrm{dm}{ }^{-3}$.

interaction between the ZnTPPS ${ }^{4-}$ molecule and the positively charged dendrimer rather than slight demetalation of ZnTPPS ${ }^{4-}{ }^{27}$

As shown in Figure 3, the analogous spectral changes were observed for the free base porphyrin under neutral and alkaline conditions, in which the Soret band at $413 \mathrm{~nm}$ were redshifted to $419 \mathrm{~nm}$ in the presence of equimolar G4 PAMAM dendrimer. A slight shoulder response around $408 \mathrm{~nm}$ at $\mathrm{pH} 7.2$ possibly relates to the H-aggregation of $\mathrm{H}_{2} \mathrm{TPPS}^{4-} .{ }^{28,}{ }^{29}$ The red-shifted fluorescence spectra at $\mathrm{pH} 7.2$ and 10.7 are also attributable to the dendrimer$\mathrm{H}_{2} \mathrm{TPPS}^{4-}$ association. The spectral results at $\mathrm{pH} 2.1$ showed rather complex features in which the UV-Vis absorption spectra indicated the appearance of new absorption peaks at 424, 483 and 699 nm (Figure 3(a), red line). The $\mathrm{H}_{2} \mathrm{TPPS}^{4-}$ molecules strongly associated with the fully protonated dendrimers could be responsible for the absorption band at $424 \mathrm{~nm}$. The other absorption bands were, however, apparently different from that of diprotonated monomers $\left(\mathrm{H}_{4} \mathrm{TPPS}^{2-}\right.$ ) (SI: Figure S1) but would be attributed to the Soret and Q bands of J-aggregates of 
$\mathrm{H}_{4} \mathrm{TPPS}^{2-}{ }^{28,30} \mathrm{~A}$ drastic decrease in the fluorescence intensity at $\mathrm{pH} 2.1$ (Figure 3(b), red line) also coincides with the formation of J-aggregates since the aggregates of the porphyrin diacids are effectively the non-fluorescent species. The aggregation behavior of the $\mathrm{H}_{4} \mathrm{TPPS}^{2-}$ monomers is strongly affected by porphyrin concentration and kinds of coexisting ions. ${ }^{31}$ In the present condition, $\mathrm{H}_{4} \mathrm{TPPS}^{2-}$ could not form the J-aggregate without adding the dendrimer. It should also be noted that the fluorescence response assigned to $\mathrm{H}_{2} \mathrm{TPPS}^{4-}$ was relatively recovered even at pH 2 by adding an excess amount of the dendrimer, i.e., [TPPS]:[dendrimer] = 1:10. The spectral results indicate that a lower protecting ability of the dendrimer for the free base porphyrin as compared to the zinc(II) complex. The porphyrins in the presence of the G2 PAMAM dendrimer exhibited spectral features similar to the G4 PAMAM dendrimer systems (SI: Figure S5).

\subsection{Ion Transfer Behavior of Dendrimer-Porphyrin Associates at the Water|DCE} Interface. Figure 4(a) shows cyclic voltammograms (CVs) in the presence of the G4 PAMAM dendrimer and ZnTPPS ${ }^{4-}$. The CVs were significantly dependent on the $\mathrm{pH}$ conditions. The net charges on the dendrimer-porphyrin associate should be reduced from the bare dendrimer because of the negative charges of the porphyrins. The voltammetric responses of the ion associates were, nevertheless, similar to those measured for the bare dendrimers (SI: Figure S6). For instance, the charge number of the G4 PAMAM dendrimer can be calculated as +126 at $\mathrm{pH}$ 2.1 due to full protonation of both 62 tertiary and 64 primary amines. The positive peak current under all $\mathrm{pH}$ conditions was almost proportional to the square root of the potential sweep rate, while the positive peak at pHs 2.1 and 10.7, buried on the gradual increase of the voltammetric responses, could not be analyzed. As reported previously, the positive peak observed around 0.40 $\mathrm{V}$ assigned as ion transfer of the G4 PAMAM dendrimer from the aqueous to the organic phases, and negative peak around $0.30 \mathrm{~V}$ as the back transfer from the organic to the aqueous phases. ${ }^{16,32}$ 

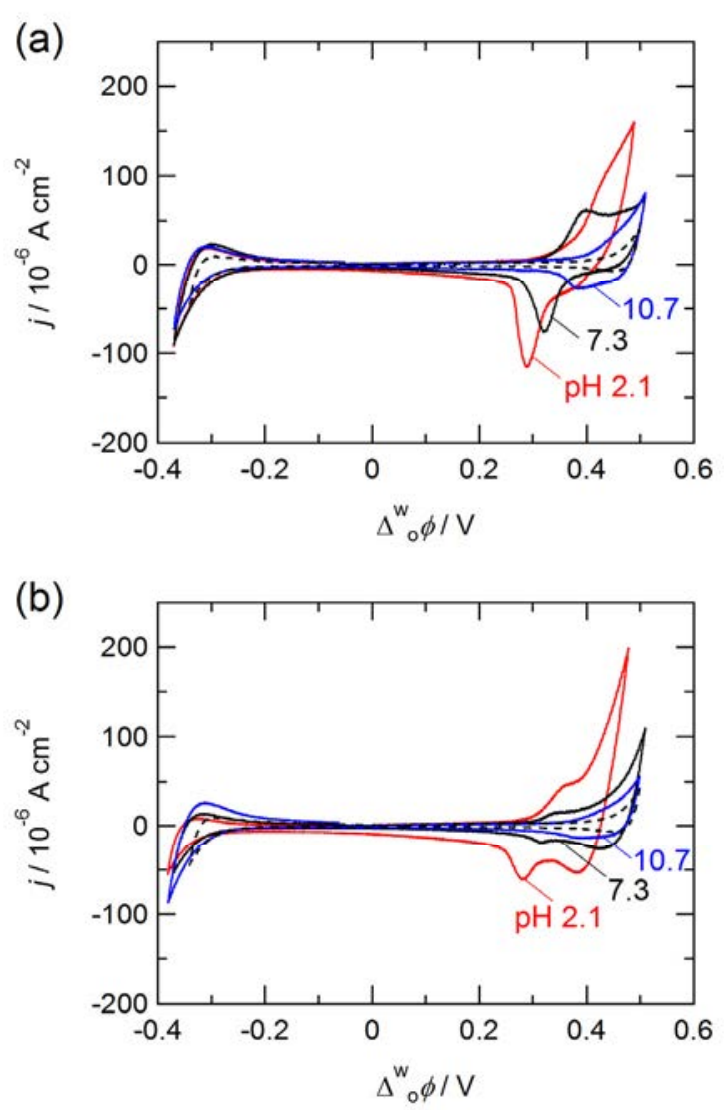

Figure 4. Typical cyclic voltammograms measured for (a) G4 and (b) G2 PAMAM dendrimers in the presence of ZnTPPS ${ }^{4-}$ at various pHs. The dashed lines are the CVs measured in the absence of ZnTPPS ${ }^{4-}$ and the dendrimer. The potential sweep rate was 50 $\mathrm{mV} \mathrm{s}^{-1}$.

In addition, a gradual increase of current response at the positive edge of the potential window was attributed to the transfer of the organic supporting electrolyte (TPFB-) facilitated by the cationic dendrimer accumulated at the interface. While the tertiary amines of the dendrimer are hardly protonated in neutral conditions, similar voltammetric responses were obtained at $\mathrm{pH}$ 7.3, in which well-defined transfer responses were measured at $0.35 \mathrm{~V}$. At $\mathrm{pH} 10.7$ where the dendrimer has almost no positive charges, the voltammetric responses were significantly 
decreased, and only the small negative responses associated with a back transfer of the dendrimer from the organic phase were found around $0.40 \mathrm{~V}$. As shown in Figure 4(b), the profile of CVs obtained for the G2 PAMAM dendrimer system was analogous to the G4 PAMAM dendrimer system. The net charge on the G2 PAMAM dendrimer with 16 amino terminal groups and 14 tertiary amines as branch points is reduced by almost quarter as compared with the G4 dendrimer under comparable conditions. Therefore, the relatively small voltammetric responses were observed at potentials close to the positive edge of potential window ( $\Delta_{0}^{\mathrm{w}} \phi>0.25 \mathrm{~V}$ ). The CVs measured for the dendrimers associated with $\mathrm{H}_{2} \mathrm{TPPS}^{4-}$ were essentially the same as the system involving ZnTPPS ${ }^{4-}$ (SI: Figure S7).

The intrinsic formal ion transfer potential $\left(\Delta_{0}^{\mathrm{w}} \phi^{\circ \prime}\right)$ of the porphyrins was determined without adding the dendrimer, respectively, as $-0.26 \mathrm{~V}$ for $\mathrm{ZnTPPS}^{4-}$ and $-0.22 \mathrm{~V}$ for $\mathrm{H}_{2} \mathrm{TPPS}^{4-}$ (SI: Figure S2) in agreement with the previous reports. ${ }^{21,33}$ The voltammetric responses of the porphyrins were, however, vanished within the polarizable potential window by the addition of the dendrimer. (SI: Figure S8). The absence of apparent transfer responses in the corresponding potential region would demonstrate that the porphyrin molecules are stably associated with the dendrimers even at the polarized water|DCE interface.

\subsection{Spectroelectrochemical Analysis of Interfacial Mechanism of Dendrimer-Porphyrin}

Associates. PMF spectroscopy was employed in order to elucidate the interfacial mechanism of the dendrimer-porphyrin associates. In this technique, the interfacial process of fluorescent ions can be selectively studied as a function of ac potential modulation superimposed on the dc bias defined as

$$
\Delta_{\mathrm{o}}^{\mathrm{w}} \phi=\Delta_{\mathrm{o}}^{\mathrm{w}} \phi_{\mathrm{dc}}+\Delta_{\mathrm{o}}^{\mathrm{w}} \phi_{\mathrm{ac}} \exp (j \omega t)
$$


where $\Delta_{0}^{\mathrm{w}} \phi_{\mathrm{ac}}$ is the amplitude of the ac potential modulation, $j$ is an imaginary number, and $\omega$ is the angular frequency. In the case that a potential-dependent process of the fluorescent ion takes place at the polarized liquid|liquid interface, the fluorescence intensity from the interfacial region is modulated with the same frequency as the potential modulation. By analyzing the phase shift and its frequency response, the ion transfer and adsorption processes are readily distinguishable. 21, 22 The PMF technique basically cannot directly be applied to the bare PAMAM dendrimer including no fluorophore. The highly fluorescent dendrimer-porphyrin associates were, however, stable even at the positively polarized water|DCE interface. The PMF analysis of the interfacial mechanism was thus successfully performed both in the dendrimer-ZnTPPS ${ }^{4-}$ and dendrimer$\mathrm{H}_{2} \mathrm{TPPS}^{4-}$ systems. The potential dependence and complex plane of the PMF responses in the presence of $1.0 \times 10^{-5} \mathrm{~mol} \mathrm{dm}^{-3}$ G4 PAMAM dendrimer and $\mathrm{ZnTPPS}^{4-}$ at $\mathrm{pH} 7.3$ are displayed in Figure 5. In the potential dependence measurements, the real $\left(\Delta F_{\mathrm{re}}\right)$ and imaginary components $\left(\Delta F_{\mathrm{im}}\right)$ of the PMF response were obtained as positive and negative signs around $0.32 \mathrm{~V}$, where the characteristic voltammetric responses were observed in CVs (c.f. Figure 4(a)). The PMF signal associated with a quasi-reversible ion transfer $\left(\Delta F_{\mathrm{t}}\right)$ is correlated with the faradaic ac current $\left(i_{\mathrm{f}, \mathrm{ac}}\right)$. In the case of the TIR excitation condition, $\Delta F_{\mathrm{t}}$ is described as ${ }^{21}$

$$
\Delta F_{\mathrm{t}}=\frac{4.606 \varepsilon \Phi_{\mathrm{f}} I_{0}}{j \omega \mathrm{zFS} \cos \psi} i_{\mathrm{f}, \mathrm{ac}}
$$

where $\varepsilon, \Phi_{\mathrm{f}}$ and $S$, respectively, are the molar absorption coefficient, the fluorescence quantum yield and interfacial area. It has been established that the real and imaginary components of the PMF signals for an ion transfer of a cationic species across the interface are expressed as positive and negative values at an ion transfer potential, respectively. ${ }^{51}$ A PMF complex plane for 

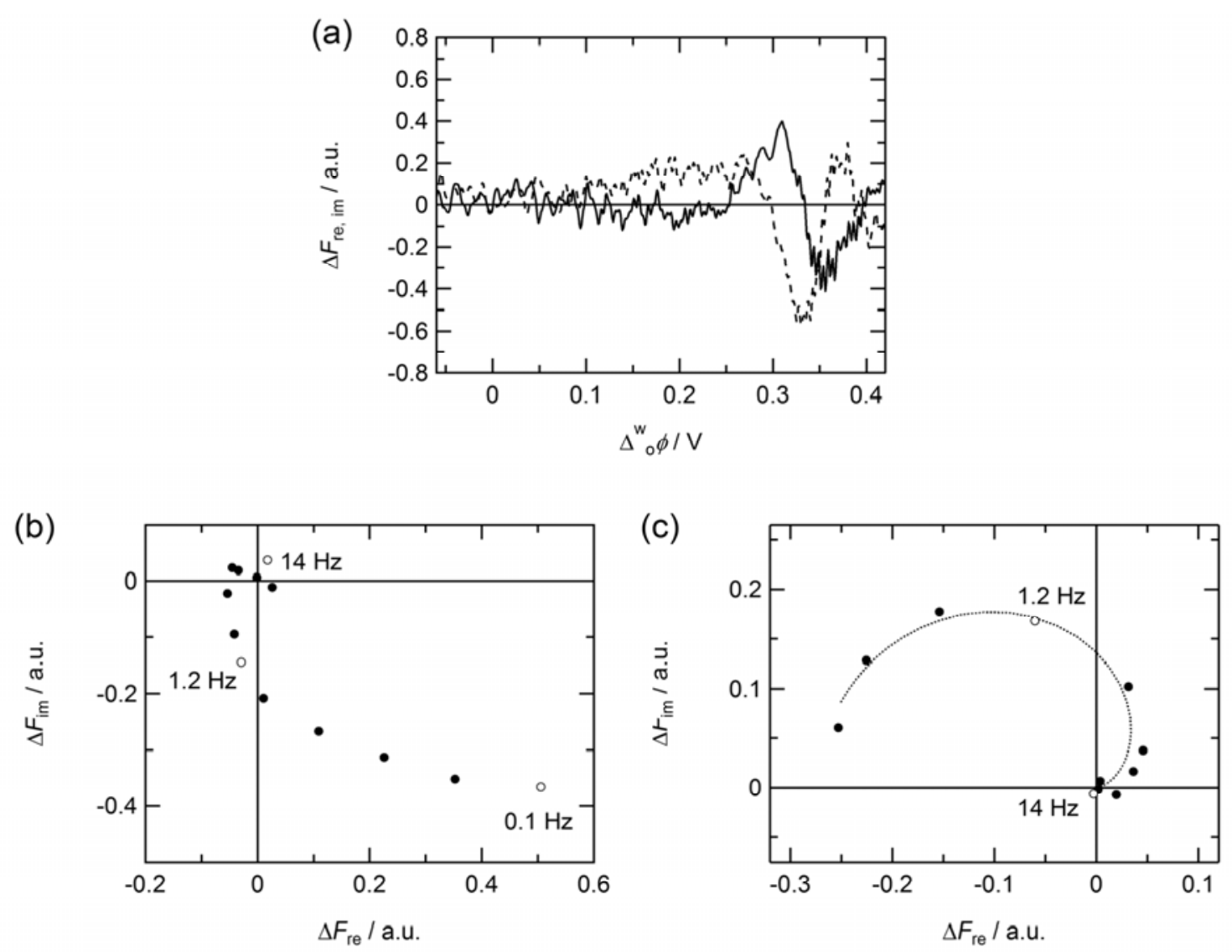

Figure 5. Dependences of the PMF responses for the G4 PAMAM dendrimer in the presence of $\mathrm{ZnTPPS}^{4-}$ at $\mathrm{pH} 7.3$ on (a) the Galvani potential difference and the potential modulation frequency at (b) 0.32 and (c) $0.37 \mathrm{~V}$. (a) The solid and dashed lines refer to the real and imaginary components, respectively. (c) The dotted line was obtained by a least-squares curve-fitting with the theoretical equation derived from eqs 3-5. The amplitude of ac potential modulation was $20 \mathrm{mV}$.

cationic species, then, appears in the fourth quadrant. On the other hand, the $180^{\circ}$ phase shift, negative real and positive imaginary components, is observed for an anionic species. The frequency dependent PMF responses measured at $0.32 \mathrm{~V}$ was expressed as a distorted linear 
response in the fourth quadrant of the complex plane (Figure 5(b)). Therefore, the PMF responses around $0.32 \mathrm{~V}$ correspond to the ion transfer of the positively charged dendrimer associated with ZnTPPS ${ }^{4-}$. The distortion of the complex plane could be considered due to the contributions of the adsorption step and the uncompensated solution resistance. ${ }^{22,} 33 \mathrm{~A}$ complicating PMF feature was also observed at potentials more positive than $0.35 \mathrm{~V}$, where the real and imaginary components were obtained mainly as negative and positive sings. The complex plane of PMF responses at $0.37 \mathrm{~V}$ exhibited a semicircle, typically obtained for an adsorption process. ${ }^{22,} 33,34$ The PMF signal associated with the adsorption $\left(\Delta F_{a}\right)$ from the aqueous phase to the interface is expressed as a function of the ac surface coverage $\left(\theta_{\mathrm{ac}}\right)^{22}$

$$
\Delta F_{\mathrm{a}}=2.303 \varepsilon \Phi_{\mathrm{f}} I_{0} \Gamma_{s} \theta_{\mathrm{ac}}
$$

where $\Gamma_{\mathrm{s}}$ is the saturated interfacial concentration. $\theta_{\mathrm{ac}}$ is described as

$$
\theta_{\mathrm{ac}}=\frac{b z F \Delta_{\mathrm{o}}^{\mathrm{w}} \phi_{\mathrm{ac}}}{R T}\left[\frac{k_{\mathrm{a}, \mathrm{dc}} \alpha c_{\mathrm{dc}}\left(1-\theta_{\mathrm{dc}}\right)-k_{\mathrm{d}, \mathrm{dc}}(\alpha-1) \theta_{\mathrm{dc}}}{k_{\mathrm{a}, \mathrm{dc}} c_{\mathrm{dc}}+k_{\mathrm{d}, \mathrm{dc}}+j \omega}\right]
$$

where $b$ is the portion of applied potential employed for adsorption process $\left(b^{\mathrm{w}}+b^{\text {org }} \leq 1\right), \alpha$ is overall transfer coefficient for adsorption process, $c_{\mathrm{dc}}$ is the bulk concentration, $\theta_{\mathrm{dc}}$ is dc surface coverage, $k_{\mathrm{a}, \mathrm{dc}}$ and $k_{\mathrm{d}, \mathrm{dc}}$ are the dc components of adsorption and desorption rate constants at given potentials, respectively. The appropriate reverse sign is applied to eq. (4) for the adsorption process from the organic phase because of an opposite potential dependence of the surface concentration. For a cationic species, the PMF complex plane of the adsorption process from the aqueous and organic sides of the interface appears in the fourth and second quadrants. The PMF response measured at $0.37 \mathrm{~V}$ was expressed as a distorted semicircle in the second quadrant 
(Figure 5(c)), demonstrating that the positively charged ion associates are transferred across the interface accompanied by the adsorption process at the organic side of the interface. The highfrequency features in the first quadrant could be associated with an attenuation of the ac potential modulation due to the uncompensated resistance across the reference electrodes. Taking into account that the ac potential can be attenuated by a magnitude proportional to the ac current and uncompensated solution resistance $\left(R_{\mathrm{u}}\right)$, the $\Delta_{0}^{\mathrm{w}} \phi_{\mathrm{ac}}$ term in eq 4 is replaced by the effective amplitude $\left(\Delta_{\mathrm{o}}^{\mathrm{w}} \phi_{\mathrm{ac}}^{\mathrm{eff}}\right)^{22,33}$

$$
\Delta_{\mathrm{o}}^{\mathrm{w}} \phi_{\mathrm{ac}}^{\mathrm{eff}}=\Delta_{\mathrm{o}}^{\mathrm{w}} \phi_{\mathrm{ac}}\left(1-\frac{R_{\mathrm{u}}}{Z_{\mathrm{T}}}\right)
$$

where the $Z_{\mathrm{T}}$ is the total impedance. As shown in Figure 5(c), the frequency responses of PMF were reproduced by the theoretical model based on eqs 3-5 (the dotted line). However, the kinetic parameters could not be determined with the acceptable error because of unknown conditional factors associated with an optical setup and "exact" charges on the dendrimerporphyrin associate.

Figure 6 shows the PMF response measured in the presence of $1.0 \times 10^{-5} \mathrm{~mol} \mathrm{dm}^{-3}$ the G4 PAMAM dendrimer and ZnTPPS ${ }^{4-}$ at various pHs. The broad PMF response around $0.10 \mathrm{~V}$ at $\mathrm{pH} 2.1$ could be due to the weak adsorption process of the dendrimer-porphyrin associates prior to the ion transfer observed at $0.33 \mathrm{~V}$. In addition, the PMF response around $0.40 \mathrm{~V}$ with an opposite sign to the ion transfer response should be the adsorption process at the organic side of the interface. No PMF responses for the adsorption process at the aqueous side were observed at pH 7.3 as described above. At pH 10.7, the PMF response for ion transfer of the ion associates alone was observed at $0.38 \mathrm{~V}$. The net charge on the ion associates dramatically decreases at $\mathrm{pH}$ 10.7. Since the potential dependent adsorption process of ionic species at polarized liquid|liquid 


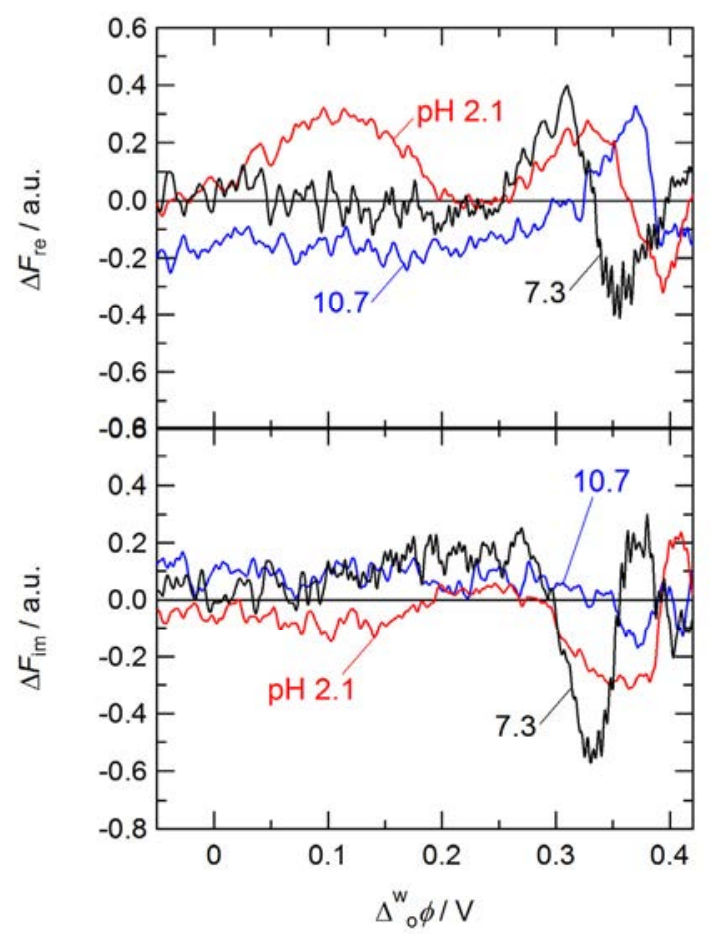

Figure 6. Potential dependence of the PMF responses for the G4 PAMAM dendrimerZnTPPS $^{4-}$ associates at various pHs. The upper and lower frames relate to the real and imaginary components, respectively. The amplitudes of ac potential modulation were $50 \mathrm{mV}$ at pH 2.1 and $20 \mathrm{mV}$ at $\mathrm{pH} 7.3$ and 10.7, respectively.

interface is substantially dominated by the bulk concentration and the Galvani potential difference, ${ }^{22,} 35$ the driving force for the adsorption process could be inefficient for less charged ions at a given potential. The disappearance of the adsorption responses at higher $\mathrm{pH}$ conditions, hence, results from the decrease in positive charges on the ion associates. The PMF results demonstrated significant differences in the interfacial mechanism of the ion associates depending on $\mathrm{pH}$.

In order to elucidate further details of the encapsulation behavior of anionic porphyrins in the dendrimers at the interface, the PMF analysis was performed in the negative potential region 

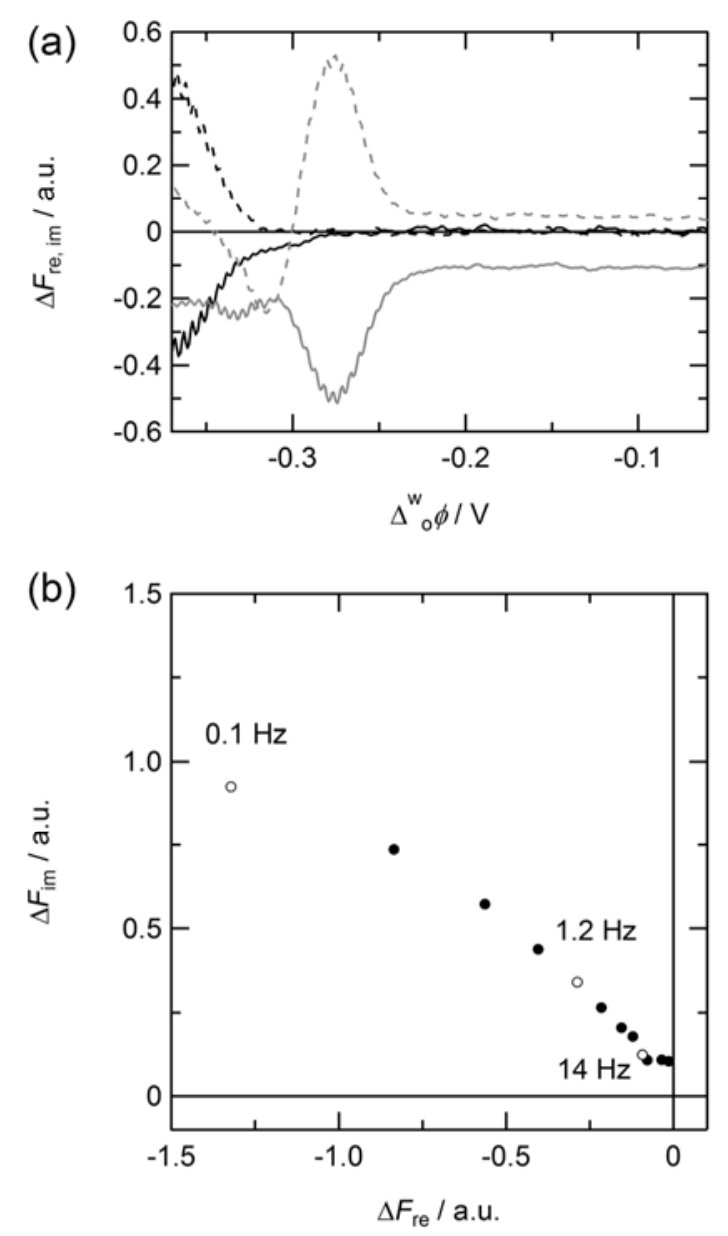

Figure 7. Dependences of the PMF response for $\mathrm{ZnTPPS}^{4-}$ in the presence of the G4 PAMAM dendrimer at $\mathrm{pH} 7.3$ on (a) the Galvani potential difference and (b) the potential modulation frequency at $-0.37 \mathrm{~V}$. (a) The gray lines refer to PMF in the absence of the dendrimer at $\mathrm{pH}$ 7.1. The solid and dashed lines refer to the real and imaginary components, respectively. The amplitude of ac potential modulation was $20 \mathrm{mV}$.

$\left(\Delta_{\mathrm{o}}^{\mathrm{w}} \phi<-0.10 \mathrm{~V}\right.$ ), where the voltammetric response for the porphyrins was effectively vanished in the presence of the dendrimer (SI: Figure S8). The buried charge transfer process in the negative edge region of the potential window was clarified by the PMF analysis. Figure 7 shows 
that the PMF responses of ZnTPPS ${ }^{4-}$ with negative real and positive imaginary components at $0.26 \mathrm{~V}$ were negatively shifted to $-0.37 \mathrm{~V}$ in the presence of the equimolar G4 PAMAM dendrimer. In addition, small PMF signals prior to the transfer response of ZnTPPS ${ }^{4-}$, associating with the adsorption process at the aqueous side of the interface, ${ }^{21,33}$ were not observed in the presence of the dendrimer. The linear PMF response in the second quadrant (Figure 7(b)) clearly indicates that the PMF responses at $-0.37 \mathrm{~V}$ are associated with the ion transfer process of the anionic ZnTPPS ${ }^{4-}$ without the adsorption step in the presence of the dendrimer. The negative shift of the ZnTPPS ${ }^{4-}$ transfer in the presence of the dendrimer from its intrinsic transfer potential at $-0.26 \mathrm{~V}$ can be considered as the result of the encapsulation of the porphyrin molecule in the dendrimer. The similar encapsulation and release behavior have been reported for the electrostatically formed ion associates between anionic anilinonaphthalenesulfonates and the carboxylate-terminated G3.5 PAMAM dendrimer at the polarized water|DCE interface. ${ }^{17}$ The shift of the transfer potential is inseparably correlated to the association energy between the dendrimer and guest ions. The negative shifts of the PMF responses associated with the transfer of the released porphyrin anions were measured in all the dendrimer-porphyrin systems examined in this work (Figure 8). It is noteworthy that the negative shift of the transfer responses of both the zinc(II) and free base porphyrins was enhanced in lower $\mathrm{pH}$ conditions, suggesting a stronger interaction of anionic porphyrins with highly protonated dendrimers. At $\mathrm{pH}$ 2.1, the PMF responses for the ion transfer of $\mathrm{ZnTPPS}^{4-}$ and $\mathrm{H}_{4} \mathrm{TPPS}^{2-}$ in the presence of the dendrimer were observed, respectively, at the negative edge $\left(\Delta_{0}^{\mathrm{w}} \phi<-0.33 \mathrm{~V}\right)$ and out of the potential window as shown in Figures 8(a,b) and (c,d). Under acidic conditions in the absence of the dendrimer, the monomeric $\mathrm{H}_{4} \mathrm{TPPS}^{2-}$ was preferentially formed in the aqueous phase, and the weak PMF signals for $\mathrm{H}_{4} \mathrm{TPPS}^{2-}$ associated with the transfer and/or adsorption at the aqueous 

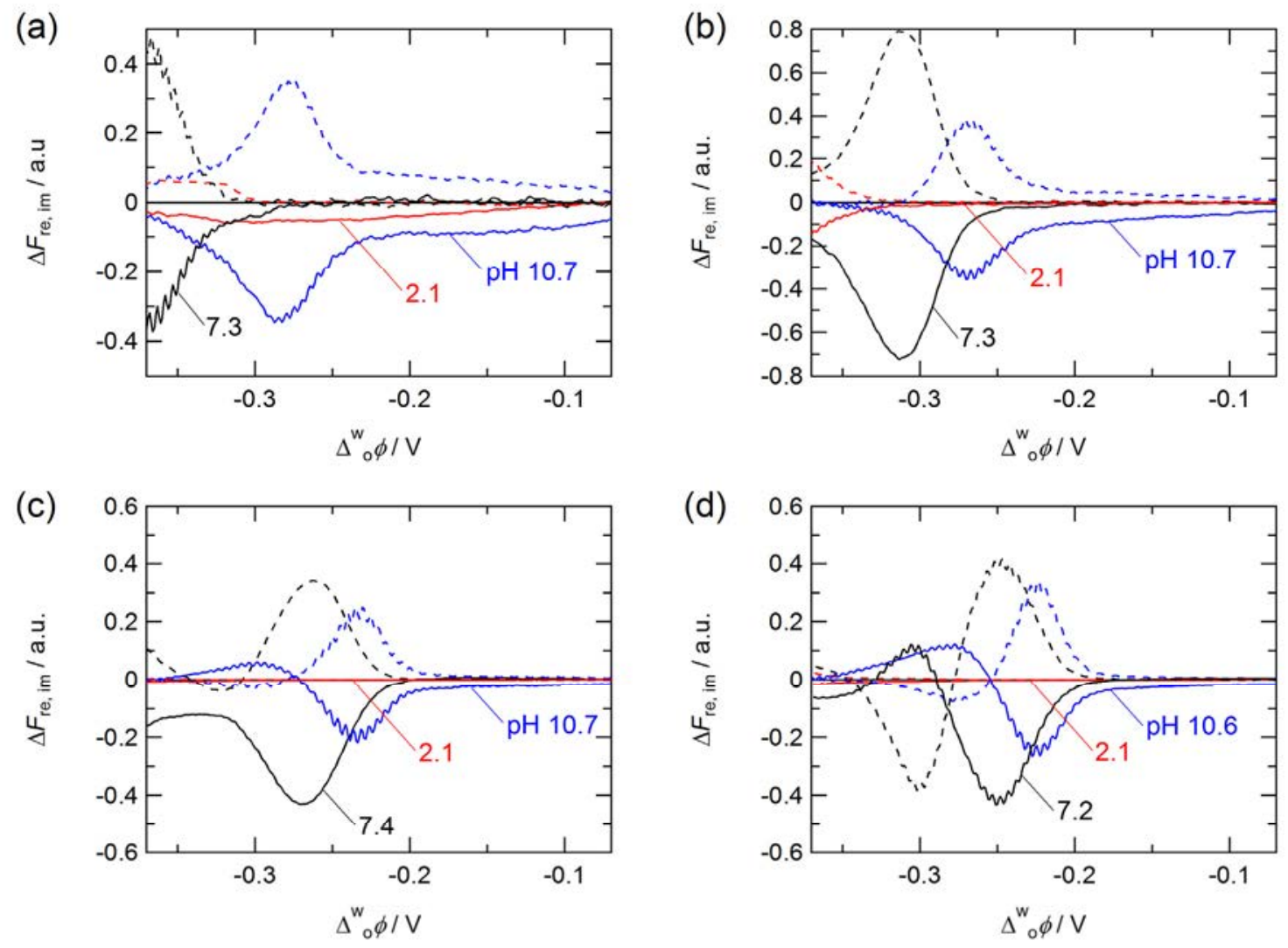

Figure 8. Potential dependences of the PMF responses of (a, b) ZnTPPS ${ }^{4-}$ and (c,d) $\mathrm{H}_{2} \mathrm{TPPS}^{4-}$ in the presence of the (a,c) G4 and (b,d) G2 PAMAM dendrimers. The solid and dashed lines refer to the real and imaginary components, respectively. The amplitudes of ac potential modulation were (a) $50 \mathrm{mV}$ at pH 2.1 and $20 \mathrm{mV}$ at pH 7.3 and 10.7, (c) $20 \mathrm{mV}$ at pH 2.1 and $10 \mathrm{mV}$ at $\mathrm{pH} 7.4$ and 10.7, respectively.

side of the interface were observed at the negative edge of the potential window ( $\Delta_{\mathrm{o}}^{\mathrm{w}} \phi<-0.3 \mathrm{~V}$ ) (SI: Figure S3). Since ZnTPPS ${ }^{4-}$ released from the dendrimer are possibly protonated via demetalation during the transfer process, the PMF signals measured for the ZnTPPS ${ }^{4-}$ systems at pH 2 might include a certain contribution of the diacid formation. The potential dependent interfacial behavior of the dendrimer-porphyrin associate at under neutral conditions can be 


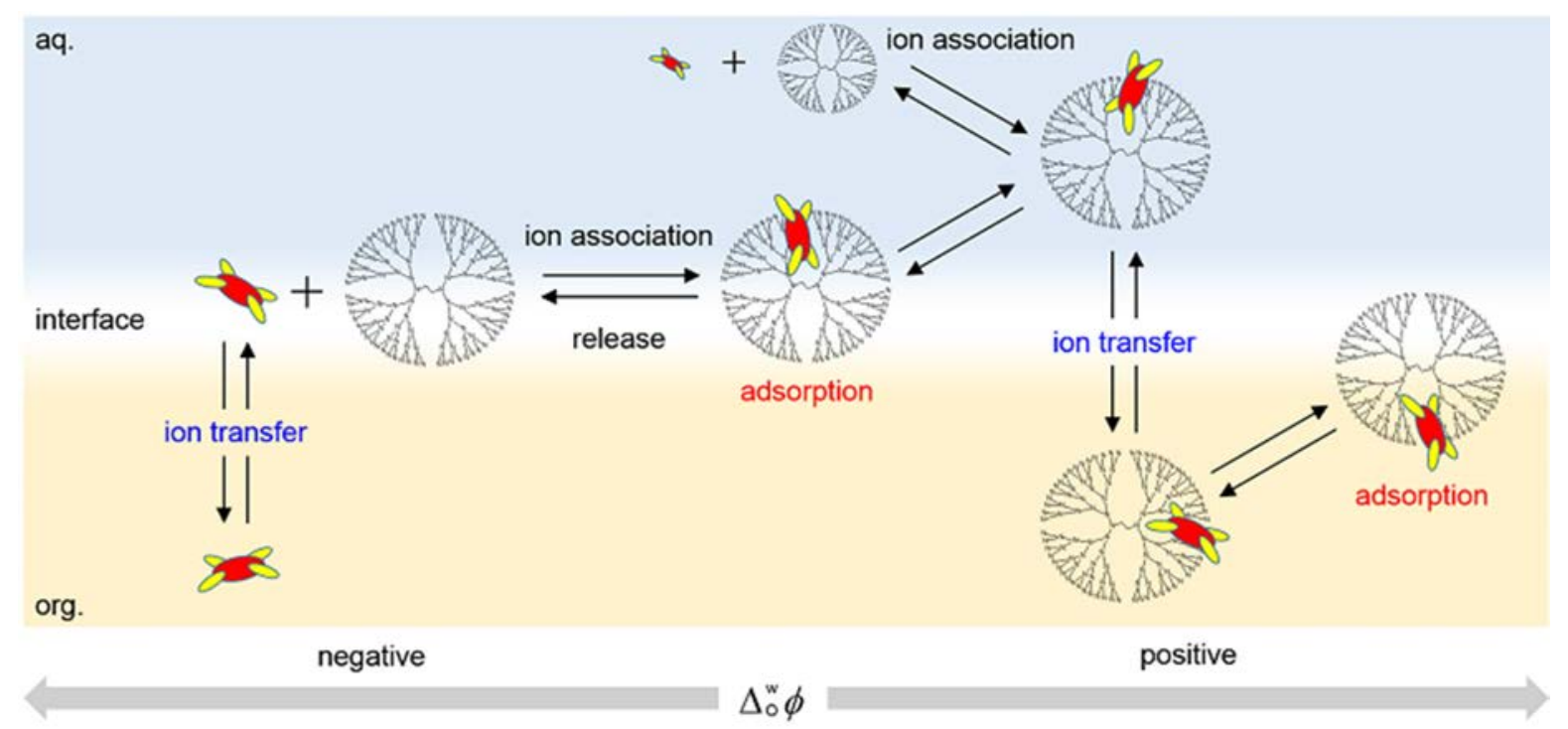

Figure 9. Schematic representation of the interfacial mechanism of the dendrimer and porphyrin under neutral conditions.

schematically shown in Figure 9. The cationic dendrimer incorporating the porphyrin molecule is transferred across the interface accompanied by the adsorption step at the positively polarized interface, while the ion transfer of the anionic porphyrin takes place at the negatively polarized interface.

The Gibbs free energy of ion association between the dendrimers and porphyrins $\left(\Delta G_{\mathrm{D} \cdots \text { porphyrin }}\right)$ can be estimated from following equation ${ }^{17}$

$$
\Delta_{\mathrm{o}}^{\mathrm{w}} \phi_{\text {porphyrin }}^{1 / 2}=\Delta_{\mathrm{o}}^{\mathrm{w}} \phi_{\text {porphyrin }}^{\mathrm{o}}-\frac{\left(\Delta G_{\mathrm{D} \cdots \text { porphyrin }}\right)_{\mathrm{pH}}}{z F}
$$

where $\Delta_{0}^{\mathrm{w}} \phi_{\text {porphyrin }}^{1 / 2}$ is the half-wave potential for the transfer of porphyrin determined from the PMF analysis, $\Delta_{0}^{\mathrm{w}} \phi_{\text {porphyrin }}^{\mathrm{o}^{\prime}}$ is the formal ion transfer potential, $z$ is the charge number of the porphyrin (-4) and $F$ is Faraday constant. The $\Delta G_{\mathrm{D} \cdots \text { porphyrin }}$ values summarized in Table 1 were 
calculated from the PMF data measured in the presence of equimolar dendrimers and porphyrins $\left(1.0 \times 10^{-5} \mathrm{~mol} \mathrm{dm}^{-3}\right)$. At $\mathrm{pH} 2.1$, the $\Delta G_{\mathrm{D} \cdots \text { porphyrin }}$ value was not precisely estimated from the PMF experiments because ZnTPPS ${ }^{4-}$ was transferred across the interface at the negative edge of the potential window and $\mathrm{H}_{2} \mathrm{TPPS}^{4-}$ formed non-fluorescent J-aggregates of the diacid species, $\left(\mathrm{H}_{4} \mathrm{TPPS}^{2-}\right)_{n}$. Assuming that the self-aggregation and specific intermolecular interaction of the porphyrin molecules encapsulated in the dendrimer are negligible in the present condition, $\Delta G_{\mathrm{D} \cdots \text { porphyrin }}$ can be used as a diagnostic criteria for the ion association stability. The lager negative values of $\Delta G_{\mathrm{D} \cdots \text { porphyrin }}$ were obtained for the ion associates with the G4 PAMAM dendrimer in comparison with the G2 PAMAM dendrimer. As discussed in Section 3.1, the G4 PAMAM dendrimer is a sufficiently large molecule with internal cavities, where the encapsulated porphyrin molecules can effectively be isolated from the aqueous phase and protected from the proton attacks. On the other hand, the porphyrin molecule cannot be accommodated in a smaller interior of the G2 PAMAM dendrimer, although the interaction with the periphery groups seems to induce the red-shift of the spectra as shown in Figure 2(a). Another finding in Table $\mathbf{1}$ is that $\Delta G_{\mathrm{D} \cdots \text { porphyrin }}$ tends to be larger negative values for the ZnTPPS $^{4-}$ system. The $\Delta G_{\mathrm{D} \cdots \text { porphyrin }}$ values therefore demonstrate the high stability of the zinc(II) porphyrin in the higher generation dendrimer. In the ion association system consisting of anilinonaphthalenesulfonates and the carboxylate-terminated G3.5 PAMAM dendrimer, it was reported that the electrostatic two-point interaction of the dianionic species (bis-ANS ${ }^{2-}$ ) enhance the ion association stability, ${ }^{17}$ e.g., the $\Delta G_{\mathrm{D} \cdots \text { bis-Ans }}$ values were $-37 \mathrm{~kJ} \mathrm{~mol}^{-1}$ at $\mathrm{pH} 2.9$ and $-6 \mathrm{~kJ}$ $\mathrm{mol}^{-1}$ at $\mathrm{pH}$ 7.6, respectively. In the present system, the molecular size and four negative charges localized on meso-sulfonatophenyl groups of the zinc(II) porphyrin are essentially the same as 
the free base porphyrin, except for the acidic condition. In the case that only electrostatic (multipoint) interactions and size effects contribute to the formation of the dendrimer-porphyrin associates, $\Delta G_{\mathrm{D} \cdots \text { porphyrin }}$ for the ZnTPPS ${ }^{4-}$ and $\mathrm{H}_{2} \mathrm{TPPS}^{4-}$ systems should be identical each other. The $\Delta G_{\mathrm{D} \cdots \text { porphyrin }}$ value for the G4 PAMAM dendrimer-ZnTPPS ${ }^{4-}$ associate at $\mathrm{pH} 7.2$ was, however, ca. $15 \mathrm{~kJ} \mathrm{~mol}^{-1}$ more negative value than that for the G4 PAMAM dendrimer$\mathrm{H}_{2}$ TPPS $^{4-}$ associate. Gentle and coworkers have reported the structural analysis of the G4 PAMAM dendrimer-copper(II) ion complexes in the aqueous solution. ${ }^{36}$ The X-ray absorption fine structure (XAFS) and NMR results indicated that the primary amine, amide and tertiary amine nitrogen atoms of the dendrimer were involved in bonding with the copper(II) ion to form five- and six-membered rings. The considerable stability enhancement for ZnTPPS ${ }^{4-}$ observed in the present study could be due to the axial coordination to the zinc(II) center of the porphyrin by amidoamine branch or tertiary amine of the dendrimer.

Table 1. The Gibbs free energies of ion association between porphyrins and dendrimers $\left(\Delta G_{\mathrm{D} \cdots \text { porphyrin }}\right.$ ) estimated from the PMF measurement ${ }^{a}$

\begin{tabular}{cccccc}
\hline & \multicolumn{4}{c}{$\Delta G_{\text {D } \cdots \text { porphyrin }} / \mathrm{kJ} \mathrm{mol}^{-1}$} \\
\cline { 2 - 3 } \cline { 5 - 6 } $\mathrm{pH}$ & $\mathrm{G} 4$ & $\mathrm{G} 2$ & & \multicolumn{2}{c}{$\mathrm{H}_{2}$ TPPS $^{4-}$} \\
\cline { 2 - 3 } \cline { 5 - 6 } 2 & $<-40$ & $<-40$ & & - & G2 \\
7 & $-30.1 \pm 0.9$ & $-18.3 \pm 0.5$ & & $-15.4 \pm 0.9$ & $-9.3 \pm 1.6$ \\
11 & $-6.3 \pm 0.7$ & $-1.4 \pm 0.7$ & & $-3.2 \pm 1.3$ & $-3.9 \pm 0.3$ \\
\hline
\end{tabular}

${ }^{a}$ The concentration of the dendrimers and the porphyrins was $1.0 \times 10^{-5} \mathrm{~mol} \mathrm{dm}^{-3}$. 


\section{Conclusions}

The ion association behavior and interfacial mechanism of the PAMAM dendrimer-anionic porphyrin associates were investigated in situ at the polarized water|DCE interface by PMF spectroscopy. The spectroelectrochemical analysis indicated that both ZnTPPS ${ }^{4-}$ and $\mathrm{H}_{2} \mathrm{TPPS}^{4-}$ favorably form the stable ion associates with the dendrimer in the aqueous solution. In particular, the G4 PAMAM dendrimer effectively inhibited the demetalation of the zinc(II) porphyrin under acidic conditions. The ion association stability was estimated from $\Delta G_{\mathrm{D} \cdots \text { porphyrin }}$ as: G4 PAMAM dendrimer-ZnTPPS ${ }^{4-}>$ G2 PAMAM dendrimer-ZnTPPS ${ }^{4-}>$ G4 PAMAM dendrimer-H2TPPS ${ }^{4-}$ > G2 PAMAM dendrimer- $\mathrm{H}_{2}$ TPPS $^{4-}$. The relatively strong interaction for ZnTPPS ${ }^{4-}$ could be result from the axial coordination to the zinc(II) center of the porphyrin molecule localized in the interior of the dendrimer. The present results clearly demonstrate that the dendrimer is capable as a molecular container as well as a protective agent for easily decomposable species. Furthermore, the encapsulation and release of a guest ion at the liquid|liquid boundary can be controlled as a function of the Galvani potential difference. The intermolecular affinity of the dendrimer could enable the development of highly functional DDS with membrane potential-sensitivity and selective separation systems.

Acknowledgment. This work was supported by Grant-in-Aids for Scientific Research (C) (no.24550097) from Japan Society for the Promotion of Science (JSPS) and Young Scientists (B) (no.21750078) from the Ministry of Education, Culture, Sports, Science and Technology of Japan (MEXT). 
Supporting Information Available: The absorption and fluorescence spectra of the monomeric porphyrins in the absence and presence of the dendrimer, CVs and ac voltammograms for the bare dendrimers, porphyrins and dendrimer-porphyrin associates, PMF data for the monomeric porphyrins. This material is available free of charge via the Internet at http://pubs.acs.org.

\section{References}

(1) Girault, H. H., Electrochemistry at Liquid-Liquid Interfaces. Electroanal. Chem. 2010, 23, $1-104$.

(2) Rao, C. N. R.; Kalyanikutty, K. P., The liquid-liquid interface as a medium to generate nanocrystalline films of inorganic materials. Acc. Chem. Res. 2008, 41, 489-499.

(3) Watarai, H.; Teramae, N.; Sawada, T., Interfacial Nanochemistry. Kluwer Academic/Plenum Publishers: New York, 2005.

(4) Volkov, A. G.; Deamer, D. W.; Tanelian, D. L.; Markin, V. S., Liquid Interfaces in Chemistry and Biology. John Wiley \& Sons: New York, 1998.

(5) Nagatani, H.; Sagara, T., Potential-modulation spectroscopy at solid/liquid and liquid/liquid interfaces. Anal. Sci. 2007, 23, 1041-1048.

(6) Fermín, D. J., Linear and Non-linear Spectroscopy at the Electrified Liquid/Liquid Interface. In Diffraction and Spectroscopic Methods in Electrochemistry, Alkire, R. C.; Kolb, D. M.; Lipkowski, J.; Ross, P. N., Eds. Wiley-VCH: Weinheim, 2006; pp 127-161.

(7) Tomalia, D. A.; Christensen, J. B.; Boas, U., Dendrimers, dendrons, and dendritic polymers : discovery, applications, and the future. Cambridge University Press: Cambridge, 2012. 
(8) Tomalia, D. A., Birth of a new macromolecular architecture: dendrimers as quantized building blocks for nanoscale synthetic polymer chemistry. Prog. Polym. Sci. 2005, 30, 294-324.

(9) Svenson, S., Dendrimers as versatile platform in drug delivery applications. Eur. J. Pharm. Biopharm. 2009, 71, 445-462.

(10) Hu, J. J.; Xu, T. W.; Cheng, Y. Y., NMR Insights into Dendrimer-Based Host Guest Systems. Chem. Rev. 2012, 112, 3856-3891.

(11) Vogtle, F.; Gestermann, S.; Hesse, R.; Schwierz, H.; Windisch, B., Functional dendrimers. Prog. Polym. Sci. 2000, 25, 987-1041.

(12) Myers, V. S.; Weir, M. G.; Carino, E. V.; Yancey, D. F.; Pande, S.; Crooks, R. M., Dendrimer-encapsulated nanoparticles: New synthetic and characterization methods and catalytic applications. Chem. Sci. 2011, 2, 1632-1646.

(13) Scott, R. W. J.; Wilson, O. M.; Crooks, R. M., Synthesis, characterization, and applications of dendrimer-encapsulated nanoparticles. J. Phys. Chem. B 2005, 109, 692-704. (14) Menjoge, A. R.; Kannan, R. M.; Tomalia, D. A., Dendrimer-based drug and imaging conjugates: design considerations for nanomedical applications. Drug. Discov. Today 2010, 15, 171-185.

(15) Pisani, M. J.; Wheate, N. J.; Keene, F. R.; Aldrich-Wright, J. R.; Collins, J. G., Anionic PAMAM dendrimers as drug delivery vehicles for transition metal-based anticancer drugs. $J$. Inorg. Biochem. 2009, 103, 373-380.

(16) Nagatani, H.; Ueno, T.; Sagara, T., Spectroelectrochemical analysis of ion-transfer and adsorption of the PAMAM dendrimer at a polarized liquid|liquid interface. Electrochim. Acta 2008, 53, 6428-6433. 
(17) Nagatani, H.; Sakamoto, T.; Torikai, T.; Sagara, T., Encapsulation of anilinonaphthalenesulfonates in carboxylate-terminated PAMAM dendrimer at the polarized water|1,2-dichloroethane interface. Langmuir 2010, 26, 17686-94.

(18) Kadish, K. M.; Smith, K. M.; Guilard, R., Handbook of Porphyrin Science. World Scientific Publishing: Singapore, 2010.

(19) Farajtabar, A.; Gharib, F.; Jamaat, P.; Safari, N., Complexation of 5,10,15,20-tetrakis(4sulfonatophenyl)porphyrin with zinc(II) ions in aqueous solution. J. Chem. Eng. Data. 2008, 53, 350-354.

(20) Wandlowski, T.; Mareček, V.; Samec, Z., Galvani Potential Scales for WaterNitrobenzene and Water-1,2-Dichloroethane Interfaces. Electrochim. Acta 1990, 35, 1173-1175. (21) Nagatani, H.; Iglesias, R. A.; Fermín, D. J.; Brevet, P. F.; Girault, H. H., Adsorption behavior of charged zinc porphyrins at the water/1,2-dichloroethane interface studied by potential modulated fluorescence spectroscopy. J. Phys. Chem. B 2000, 104, 6869-6876.

(22) Nagatani, H.; Fermín, D. J.; Girault, H. H., A kinetic model for adsorption and transfer of ionic species at polarized liquid|liquid interfaces as studied by potential modulated fluorescence spectroscopy. J. Phys. Chem. B 2001, 105, 9463-9473.

(23) Maiti, P. K.; Cagin, T.; Lin, S. T.; Goddard III, W. A., Effect of solvent and pH on the structure of PAMAM dendrimers. Macromolecules 2005, 38, 979-991.

(24) Da Costa, V. C. P.; Ribeiro, A. C. F.; Sobral, A. J. F. N.; Lobo, V. M. M.; Annunziata, O.; Santos, C. I. A. V.; Willis, S. A.; Price, W. S.; Esteso, M. A., Mutual and self-diffusion of charged porphyrines in aqueous solutions. J. Chem. Thermodyn. 2012, 47, 312-319. 
(25) Leisner, D.; Imae, T., Polyelectrolyte behavior of an interpolyelectrolyte complex formed in aqueous solution of a charged dendrimer and sodium poly(L-glutamate). J. Phys. Chem. B 2003, 107, 13158-13167.

(26) Wang, X.; Zhao, L.; Ma, R.; An, Y.; Shi, L., Stability enhancement of ZnTPPS in acidic aqueous solutions by polymeric micelles. Chem. Commun. 2010, 46, 6560-6562.

(27) Paulo, P. M. R.; Costa, S. M. B., Interactions in noncovalent PAMAM/TMPyP systems studied by fluorescence spectroscopy. J. Phys. Chem. B 2005, 109, 13928-13940.

(28) Paulo, P. M. R.; Costa, S. M. B., Non-covalent dendrimer-porphyrin interactions: The intermediacy of H-aggregates? Photochem. Photobiol. Sci. 2003, 2, 597-604.

(29) Paulo, P. M. R.; Gronheid, R.; De Schryver, F. C.; Costa, S. M. B., Porphyrin-Dendrimer Assemblies Studied by Electronic Absorption Spectra and Time-Resolved Fluorescence.

Macromolecules 2003, 36, 9135-9144.

(30) Kubát, P.; Lang, K.; Zelinger, Z., Interaction of porphyrins with PAMAM dendrimers in aqueous solution. J. Mol. Liq. 2007, 131-132, 200-205.

(31) Choi, M. Y.; Pollard, J. A.; Webb, M. A.; McHale, J. L., Counterion-dependent excitonic spectra of tetra(p-carboxyphenyl)porphyrin aggregates in acidic aqueous solution. J. Am. Chem. Soc. 2003, $125,810-820$.

(32) Berduque, A.; Scanlon, M. D.; Collins, C. J.; Arrigan, D. W. M., Electrochemistry of Non-Redox-Active Poly(propylenimine) and Poly(amidoamine) Dendrimers at Liquid-Liquid Interfaces. Langmuir 2007, 23, 7356-7364.

(33) Nagatani, H.; Ozeki, T.; Osakai, T., Direct spectroelectrochemical observation of interfacial species at the polarized water/1,2-dichloroethane interface by ac potential modulation technique. J. Electroanal. Chem. 2006, 588, 99-105. 
(34) Osakai, T.; Yamada, H.; Nagatani, H.; Sagara, T., Potential-Dependent Adsorption of Amphoteric Rhodamine Dyes at the Oil/Water Interface as Studied by Potential-Modulated Fluorescence Spectroscopy. J. Phys. Chem. C 2007, 111, 9480-9487.

(35) Kakiuchi, T., Potential-dependent adsorption and partitioning of ionic components at a liquid|liquid interface. J. Electroanal. Chem. 2001, 496, 137-142.

(36) Tran, M. L.; Gahan, L. R.; Gentle, I. R., Structural studies of copper(II)-amine terminated dendrimer complexes by EXAFS. J. Phys. Chem. B 2004, 108, 20130-20136. 
table of contents only

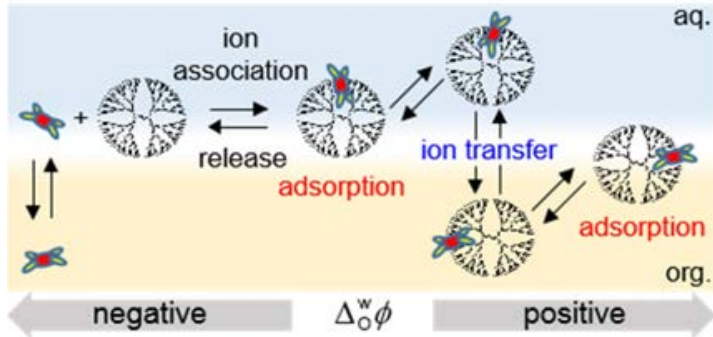

